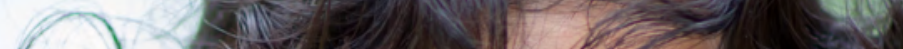

How do early adverse experiences increase the risk for mental health problems?

\author{
By Dr. Jessica Edwards
}

Early adverse experiences can predict a variety of mental health problems later in life, from anxiety to rule breaking behaviour and impulsivity. ${ }^{1}$ However, the underlying pathways by which different types of early adverse experiences, increase the risk for mental health problems, are less clear.

One model - the Dimensional Model of Adversity and Psychopathology (DMAP) - hypothesizes that deprivation and threat, impact on psychopathology, through distinct pathways. ${ }^{2}$ Indeed, initial tests of this model showed that early childhood deprivation, but not threat, was associated with late adolescent psychopathology, via language ability in early adolescence. ${ }^{3} \mathrm{It}$ is, however, still unknown whether language ability may link deprivation with psychopathology risk at earlier stages of development.

To address this knowledge gap, Adam Bryant Miller, Laura Machlin and colleagues in the US evaluated a theoretical model that linked early deprivation exposure with psychopathology via language ability. To do so, they analysed data from $>2,000$ youths enrolled in the Fragile Families and Child Wellbeing Study. They collected data on deprivation and threat (using various indicators) at ages 1 and 3 years and assessed language abilities at age 5 years. Finally, they collected and examined indicators for psychopathology at ages 5, 9 and 15 years. Consistent with the DMAP model, Miller et al. found that the impact of deprivation (from birth to age 3 years), but not threat, on psychopathology, from early childhood to adolescence can be explained, in part, by early childhood language ability.

"These findings highlight language as a potentially unique pathway for deprivation exposure and mental health problems", says Miller. "Specifically, children who have had high levels of deprivation, such as neglected children or children of parents who don't have the time and resources to invest in their development at home, may benefit from educational interventions to prevent onset of mental health problems across childhood". Additional studies that include a more diverse sample and that harness more robust measures of cognitive ability and test for other potential confounders (such as stress exposure) are now warranted.

\author{
Referring to: \\ Miller, A.B., Machlin, L., \\ McLaughlin, K.A. \& Sheridan, \\ M.A. (2020), Deprivation and \\ psychopathology in the Fragile \\ Families Study: A 15-year \\ longitudinal investigation. J. \\ Child Psychol. Psychiatr. doi: \\ 10.1111/jcpp.13260.
}

See also:

\section{https://fragilefamilies. princeton.edu/}

\section{References:}

${ }^{1}$ Keyes, K.M. et al. (2012). Childhood maltreatment and the structure of common psychiatric disorders. Br. J. Psychiatry. 200, 107-115. doi: 10.1192/bjp.bp.111.093062.

${ }^{2}$ Sheridan, M.A. et al. (2014). Dimensions of early experience and neural development: Deprivation and threat. Trends Cogn. Sci. 18, 580-585. doi: 10.1016/j.tics.2014.09.001.

${ }^{3}$ Miller, A.B. et al. (2018). Dimensions of deprivation and threat, psychopathology, and potential mediators: A multiyear longitudinal analysis. J. Abnorm. Psychol. 127, 160-170. doi: 10.1037/abnooo0331 